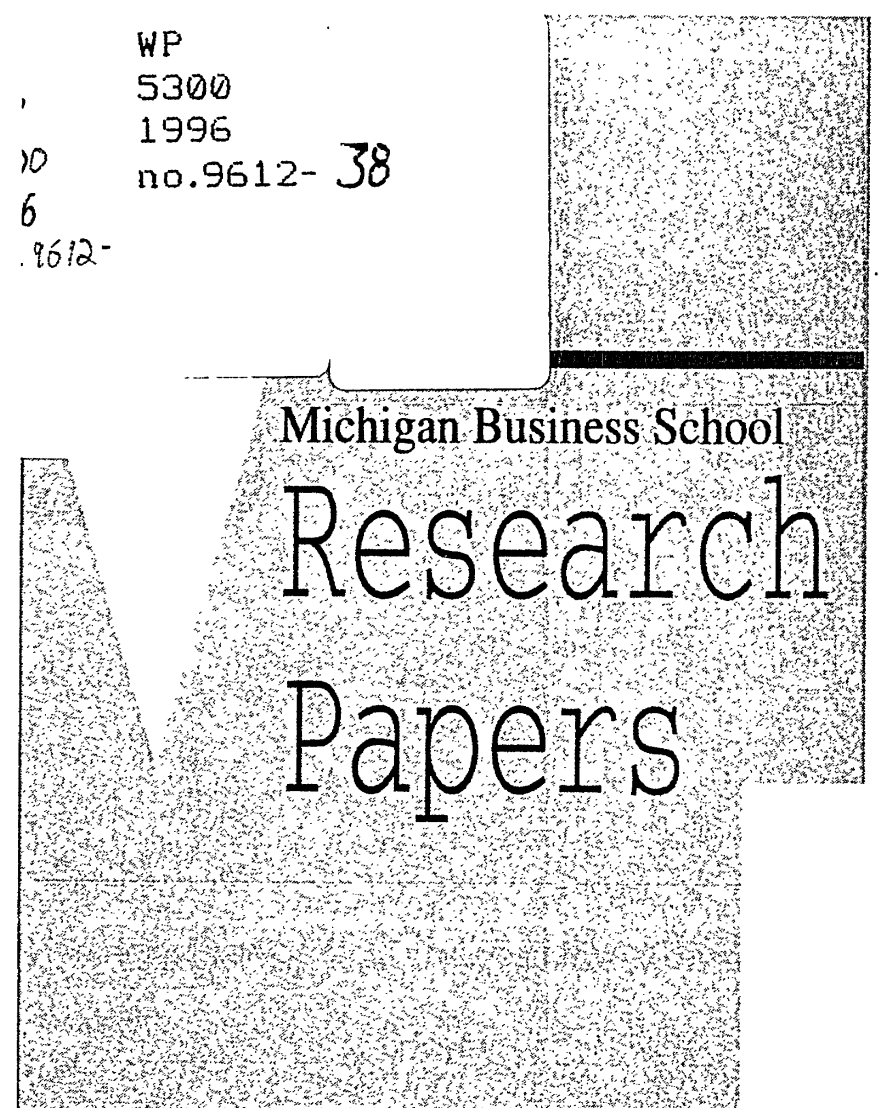

Bayesian Nonparametric and Covariate Analysis of Failure Time Data

WORKING PAPER \#96 12-38

PURUSHOTTAM W. LAUD

Medical College of Wisconsin

AN D

PAUL DAMIEN

UNIVERSITY OF MICHIGAN BUSINESS SCHOOL AND

ADRIAN F.M. SMITH

I MPERIAL COLLEGE, LONDON, UK 
UNIVERSITY OF MICHIGAN BUSINESS SCHOOL

Bayesian Nonparametric and Covariate Analysis of Failure Time Data

WORKING PAPER \#9612-38

PURUSHOTTAM W. LAUD Medical College of Wisconsin AND

PAUL DAMIEN

UNIVERSITY OF MICHIGAN BUSINESS SCHOOL AN D

ADRIAN F.M. SMITH

IMPERIAL COLLEGE, LONDON, UK 


\title{
Bayesian nonparametric and covariate analysis of failure time data
}

\author{
By Purushottam W. Laud \\ Dirision of Biostatistics. Medical College of Wisconsin. Milwaukiee. WI 53226-0.509. \\ U.S.A. \\ Paul Damien \\ Department of Statistics and Wanagement. Science. Lniversity of Wichigan. Ann Arbor. \\ MI 48109-1234, U.S.A. \\ and Adrian F.M. Smith \\ Department of Mathematics. Imperial College. London SW7 2BZ. U.K.
}

\section{SUMMARY}

A Bayesian analysis of the semi-parametric regression model of Cox (1972) is given. The cumulative hazard function is modelled as a beta process. The posterior distribution of the regression parameters and the survival function are obtained using a combination of recent Monte Carlo methods. An illustrative analysis within the context of survival time data is given.

Some key words: Infinitely Divisible Distributions: Laplace Transform: Beta Process: Hazard Function: Latent Variables; Cox Model: Gibbs Sampler.

\section{Introduction}

Cox (1972) proposed a model for survival time data in the presence of covariates. To state it. let $T$ represent the time to the event of interest for an individual and $\mathbf{z}=\left(\tilde{z}_{1} \ldots \ldots, z_{p}\right)^{\prime}$ the vector of corariates. Then the survival distribution of $T$ is taken to be

$$
P(T>t \mid \mathbf{z})=\exp \left\{-H(t) \exp \left(\mathbf{z}^{\prime} 3\right)\right\}
$$

where $H(t)=\int_{0}^{t} h(s) d s$ is the baseline cumulative hazard function (CHF), $h($.$) the cor-$ responding hazard rate and $\beta=\left(\beta_{1}, \ldots, \beta_{p}\right)^{\prime}$ is the vector of regression coefficients. This 
model is often called the proportional hazards model since the ratio of hazard rates of two individuals with differing $z \mathrm{~s}$ is coustant in time. Its use and success are now so widespreadl [see. for example. Klein anr . Moeschberger (1996) and the references therein] as to make it the primary tool in the analysis of lifetime data.

In his original article Cox used a factor of the full likelihood. which he later justified and termed partial likelihood in Cox (19i5). to estimate 3. Kalbfleisch (19i8) considered a Bavesian analysis using a gamma process prior on the logarithm of the baseline surviral function and Clayton (1991) discussed a . Monte Carlo method for related frailty models. Designed for use in various survival analysis problems, the beta process was adranced by Hjort (1990) as a prior on the space of CHF's. He also briefly treated the C'ox model and gave some descriptions of the resulting posterior distributions.

In this paper. we follow Hjort (1990) and model the cumulative hazard using a beta process. We give a full Bavesian solution in that the posterior distributions of the baseline CHF $H(t)$ and the regression coefficient vector 3 are obtained. This is accomplished by using and extending a combination of Monte Carlo methods described in Smith and Roberts (1993). Damien et.al (1995) and Walker (1995). By straightforward transformations, samples obtained from the full posterior distribution also allow inference for other relevant quantities such as the baseline survival function. the relative risk and surrival probabilities at given values of covariates and times.

Section II below describes the use of the beta process with the Cox model and the attendant posterior results available from Hjort (1990). In Section III, we develop the computational form of the model and give details for its implementation. Section IV provides an illustrative analysis of the leukemia data previously analysed, using Bayesian methods. by Kaalbfleisch (1978). Some extensions are discussed in Section V.

\section{Cox Model with Beta Process Prior}

Much of nonparametric Bayesian inference has proceeded by modelling the unknown cumulative distribution function $(\mathrm{CDF})$ as a stochastic process. One of the exceptions appears in Hjort (1990) where an alternative is introduced by placing a (beta) stochastic process on the space of cumulative hazard functions. This has several attractive features: it offers a broader class of models in the context of life history data; there is an accessible interpretation of the prior process in terms of hazard functions; and it provides a natural generalisation of neutral to the right processes. such as the Dirichlet (Ferguson. 1973).

In the discussion that follows, we will consider the general time-continuous version of the beta process. Let $T$ be a random variable with $C D F F(t)=\operatorname{Pr}(T \leq t)$ on $[0 . x)$ 
and $F(0)=0$. The CHF for $F$ or $T$ is a nommegative. nondecreasing. right continuons function $t$ on $[0 . x)$. As in Hjort (1990) we use the stmbol $A$ in three different ways : $A[a, b) . A(t)$ and.$A\{t\}$ denote the increment of the function $A$ in the interval $[a . b)$. the value of the-function $t$ at a point $t$. and the increment of the function $t$ at $t$, respectively: Now let $t$ be such that it satisfies

$$
d . A(s)=A[s . s+d s)=\operatorname{Pr}\{T \in[s . s+d s) \mid T \geq s\}=d F(s) / F[s . x)
$$

so that

$$
f(a, b)=\int_{[a, b)} \frac{d F(s)}{F[s \cdot x)} .
$$

Recorering $F$ from $A$ requires product integrals (Gill and Johansen. 1990) as

$$
F(t)=1-\prod_{[0 . t]}\{1-d A(s)\} . t \geq 0 .
$$

If $F$ is continuous, this can be shown to $r^{\prime} \cdot r e$ to the usual formula

$$
A(t)=-\log (1-F(t))
$$

In general. however. $F(t)$ does not equal $1-\exp \{-t(t)\}$. As explicated in Anclersen et al.(199.3), this definition of the cumulative hazard function unifies the treatment of discrete. continuous and mixed random variables while retaining the appropriate meaning of hazard as used in the actuarial and survival analysis literature.

While we omit the mathematical details of its construction. the beta process has independent increments that, infinitesimally speaking, have beta distributions. More precisely: it is a Lévy process with Lévy measure concentrated on $[0,1]$. Using this process as a prior on the space of cumulative hazard functions, the posterior distribution is. in the absence of covariates, again a beta process. Relevant definitions and results are stated in the Appendix. Full Bayesian inference using the Gibbs sampler for this situation is given in Damien et al (1996).

To include covariates as prescribed in the Cox model (1). let $A_{i}$ and $z_{i}$ denote the cumulative hazard function and the covariate vector for individual $i, i=1, \cdots, n$. Then.

$$
1-d A_{i}(s)=\{1-d A(s)\}^{e x p\left(z_{i}^{\prime}(3)\right.}
$$

Suppose $X_{1} \cdots \ldots X_{n}$ are the times to the event of interest for the $n$ individuals and the data are in the form $t_{i}=\min \left(X_{i}, c_{i}\right), \delta_{i}=I\left(X_{i} \leq c_{i}\right)$, where $I$ denotes the indicator function. The $c_{i}$ s here are censoring times assumed to be independent of the event times. 
Now the discussion in Section 6 of Hjort (1990) leads to the following likelihoorl for .t and 3 :

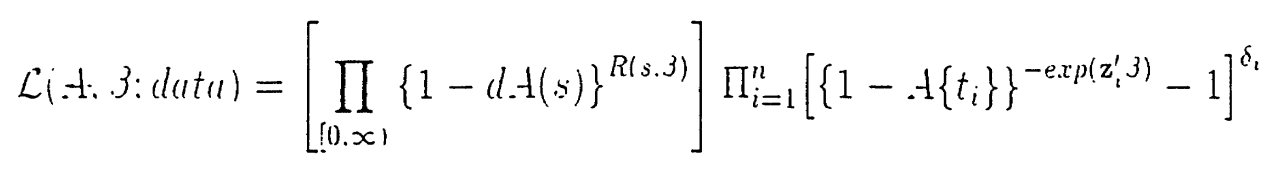

where $R(s .3)=\sum_{j=1}^{n} \exp \left(\mathbf{z}_{i}^{\prime}, 3\right) I\left(t_{j} \geq s\right)$. Lsing a beta process prior $A \sim \operatorname{beta}\left(c(.) . t_{0}().\right)$ for $A$ (see Appendix for notation) and an independent prior. for 3 . the conditional posterior of $A$ given 3 and the data is a beta process between points where actnal erent times are observed. At such event times. there are positive jumps in $t$ with distributions

$$
f_{A\{s\}}(u) \times u^{-1}(1-u)^{c(s)+R(s .3)-d(s .3)-1} \Pi_{i=1}^{n}\left\{1-d_{i}(s)(1-u)^{e . x p\left(\mathbf{z}_{i}^{\prime} 3\right)}\right\}
$$

where $d_{i}(s)=I\left(t_{i}=s \cdot \delta_{i}=1\right)$ and $d(s, 3)=\sum_{j=1}^{n} d_{i}(s) \exp \left(\mathbf{z}_{i}^{\prime} 3\right)$. At prior specification for 3 and the likelihood in (2) above suffice to vield a conditional posterior for 3 giren A. In the next section we employ these two conditionals and several auxiliary variables to develop a Gibbs sampler.

\section{The Computational Model}

Let $\Delta=\left(\Delta_{1} \cdots \Delta_{.}\right)$denote increments in the $A(\cdot)$ process between the $t_{i}$ is; i.e.. the gricl is taken to be all distinct censoring or event times in the data. Call these time points $s_{1}<s_{2}<\cdots<s_{1}$. among which are event times $x_{1}<x_{2}<\cdots<x_{m}$ occuring with multiplicities $k_{1}, k_{2}, \cdots, k_{m}$ repectively. Let $U=\left(U_{1} \cdots U_{m}\right)$ stand for the jumps in $A(\cdot)$ at these $x$ 's. Finally, let $i(j)$ be the index of the $j^{\text {th }}$ event observation at $x_{i}, j=1, \cdots, k_{\text {i }}$ in some fixed order for each $i=1, \cdots, m$. This means that $i(j)$ is a unique number between 1 and $n$ such that $t_{i(j)}=x_{i}$ and $\delta_{i(j)}=1$.

With this notation, the Gibbs sampler proceeds by simulating from [ $\triangle . L i \mid 3$, data] and $[3 \mid\lrcorner . L^{\prime}$, data $]$. As outlined in the previous section, $[\Delta \mid \beta$, data $]$ is the distribution of the increments of a beta process with parameters $\{c(\cdot)+R(\cdot, \beta)\}$ and $\int_{0}^{(\cdot)} \frac{c(s) d \cdot A_{0}(s)}{c(s)+R(s, 3)}$. The components of $\Delta$ are independent. each with an infinitely divisible distribution. Simulation of these is detailed in Damien et al.(1996). Moreover, $U$ and $\Delta$ are independent, $U^{*}$ haring independent components with distributions

$$
f\left(u_{i}\right) x u_{i}^{-1}\left(1-u_{i}\right)^{c\left(x_{i}\right)+R\left(x_{i}, 3\right)-\left\{\sum_{j=1}^{k_{2}} \exp \left(z_{i(j)}^{\prime}\right)^{3)}\right\}-1} \Pi_{j=1}^{k_{i}}\left\{1-\left(1-u_{i}\right)^{\exp \left(z_{i(j)}^{\prime}\right)^{3)}}\right\} .
$$

Simulation from such a distribution can be effected using auxiliary variables. Transforming $u$ to $u=-\log (1-u)$. the task is to simulate from

$$
[u] \times \exp p\left(-a l^{\prime}\right)(1-\exp (-v))^{-1} \Pi_{i=1}^{k}\left(1-\exp \left(-b_{i} v\right)\right) \text {. }
$$


With $y$ a geometric variate and $u_{i}$ truncated exponentials given by

$$
\left[y \mid \iota^{\prime}\right]=\left(1-\exp \left(-\iota^{\prime}\right)\right) \exp \left(-\imath^{\prime} y\right) . \quad y=0.1 . \cdots
$$

and

$$
\left[u_{i} \mid \iota^{\prime}\right]=b_{i} l^{\prime} \exp \left(-b_{i} u^{\prime} u_{i}\right)\left(1-\exp \left(-b_{i} l^{\prime}\right)\right)^{-1} I_{(0.1)}\left(u_{\imath}\right)
$$

we get

$$
\left[u^{\prime} \mid u^{\prime}, y\right] \times u^{k} \exp \left\{-u^{\prime}\left(a+y+\sum_{i=1}^{k} b_{\imath} u_{i}^{\prime}\right)\right\}
$$

which is a gamma variate.

It only remains to simulate $3 \mid \Delta . \zeta^{\prime}$. clata. Adapting the likelihood (2) to the notation of the current section yields

$$
\begin{aligned}
& {\left[.3 \mid \Delta . L^{*} \text {. data }\right] \quad x \quad[3]\left\{\Pi_{i=1}^{*}\left(1-\Delta_{i}\right)^{R\left(s_{2}, 3\right)}\right\}} \\
& \Pi_{l=1}^{m}\left(1-u_{i}\right)^{R\left(x_{i}, 3\right)-d\left(x_{i}, 3\right)} \Pi_{j=1}^{k_{i}}\left\{1-\left(1-u_{i}\right)^{\left.\exp \left(\mathbf{z}_{\imath(\jmath)}^{\prime}\right)^{3}\right)}\right\}
\end{aligned}
$$

where [.3] is the prior distribution of the regression coefficients. Using $v_{i}=-\log \left(1-u_{i}\right)$ and incorporating the conditionals of the auxiliary variables $y_{i}, w_{i j}, i=1 . \cdots, m . j=$ 1. $\cdots, k_{i}$. we arrive at

$$
\begin{aligned}
& {\left[3 \mid \Delta . Y_{Y}: W, \text { data }\right] \times[3] \exp \left[\sum_{i=1}^{N} R\left(s_{i}, \beta\right) \log \left(1-\Delta_{i}\right)\right.} \\
& \left.\quad-\sum_{i=1}^{m} v_{i}\left\{R\left(x_{i}, 3\right)-\sum_{j=1}^{k_{i}}\left(1-w_{i j}\right) \exp \left(\mathbf{z}_{i(j)}^{\prime} ; 3\right)\right\}+\sum_{i=1}^{m} \sum_{j=1}^{k_{i}} \mathbf{z}_{i(j)}^{\prime}, 3\right] .
\end{aligned}
$$

It is straightforward to show that this is logconcave in each component of 3 if the prior is so. leading to efficient simulation of $\beta$ by the algorithm of Gilks and Wild (1992). To summarize. we use

$$
\begin{aligned}
& {[Y \mid \beta, \Delta, V, W, \text { data }] \propto \text { Independent Geometrics }} \\
& [W \mid 3 .\lrcorner . V, Y, \text { data }] \propto \text { Independent Truncated Exponentials } \\
& [V \mid ; 3 .\lrcorner, W, Y, \text { data }] \propto \text { Independent Gammas } \\
& {[\triangle \mid 3 . V, W, Y, \text { datal }] \propto \text { Independent Beta Process Increments }} \\
& {[3 \mid \triangle . V . W, Y \text {.data }] \propto \text { Componentwise Logconcave }}
\end{aligned}
$$

as the full conditionals in a Gibbs sampler. 
Table 1: Leulemia Remission Time Data

\begin{tabular}{|c|c|}
\hline Group 0 (dring) & 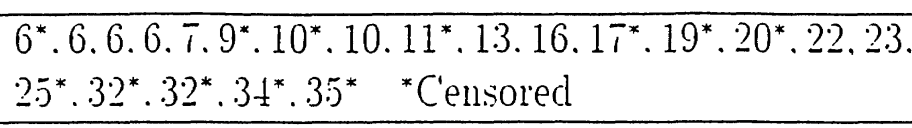 \\
\hline Group 1 (placebo) & $1.1 .2 .2 .3 .4 .4 .5 .5 .8 .8 .8 .11 .11 .12 .12 .15 .1 \% .22 .23$ \\
\hline
\end{tabular}

\section{Illustrative Analysis}

To demonstrate the trpes of inference one can make using the techniques of this article. we consider the leukemia data analyzed by Cox (19-2) and Kalbfleisch (19-8). aniong others. These data. listed in Table 1 as reported by these authors, consist of remission times (in weeks) of leukemia patients assigned to treatment with a drug or a placebo during remission maintenance therapy. Although Andersen et al.(1993) point out that the data actually consisted of 21 pairs matched by status of initial remission attained. we have chosen to disregard this aspect here in view of the present purposes of illustration and comparison.

In the analyses below. the prior distribution for $\beta$ is taken to be normal with mean 1.5 and standard deviation 5.0. Runs with varying means in the range -5.0 to 10.0 gare similar results not reported here. The prior for the baseline cumulative hazard function is a beta process with $A_{0}(t)=0.05 t$ and $c(t)=k e^{-0.05 t}$. This simple choice corresponds to a prior mean survival function that is exponential with mean 20 while the prior rariability can be controlled via the number $k$. Our illustrative calculations use a wide range for $k$ from 0.01 to 100.0. The beta process, however, admits a much richer expression of prior opinion as designed in Hjort (1990) and demonstrated in Damien et al.(1996). One can specify different time-dependent degrees of prior variance in $A(t)$ since $c(t)$ can be interpreted as the number at risk at $t$ in an imagined prior sample. Such flexibility is especially welcome in constructing prior specifications from past data sets and reported studies.

With these priors and the data in Table 1 the Gibbs sampler of the previous section was implemented and run with each setting of $k$, saving 2000 iterations after discarding the first 3000. The results for $\beta$ are summarized in Table 2. Values of $k$ near zero represent little prior input whereas large values correspond to a strong prior belief that the baseline hazard is that of an exponential distribution with mean 20. These posterior summaries are remarkably stable over a large range of $k$, a phenomenon observed also by Kalbfleisch $(19 i 8)$ regarding the posterior mode and an approximation to the posterior standard deviation when using a gamma process prior. In Figure 1(a) we show the 
Table 2: Posterior Summaries for 3

\begin{tabular}{|c||ccccc|}
\hline \multirow{2}{*}{ Summary } & \multicolumn{5}{c|}{$k$} \\
\cline { 2 - 6 } & 0.01 & 0.10 & 1.0 & 10.0 & 100.0 \\
\hline \hline Mean & 1.69 & 1.71 & 1.71. & $1.6 \overline{1}$ & 1.62 \\
Standard deriation & 0.438 & 0.421 & 0.42 .2 & 0.419 & 0.381 \\
Central 90\% limits & $(0.99 .2 .43)$ & $(1.06 .2 .44)$ & $(1.02 .2 .42)$ & $(0.98 .2 .36)$ & $(0.97 .2 .25)$ \\
\hline
\end{tabular}

posterior distributions of 3 .

For comparison with results based on maximizing the partial likelihood. calculations were carried out using four different methods including those advanced by Efron (19Ti) and Falbfleisch and Prentice (1980) to accomodate tied observations. Details of these are given. for example. in Section 8.3 of Kilein and Moeschberger (1996). Estimates of 3 raried from 1.51 with the method of Breslow (19it) to 1.63 by that of Cox (19T2). The discrepancy is about $28 \%$ of the standard error. In contrast, the Bayesian analysis in this paper uses the full likelihood and calculates the appropriate posterior, with or without multiplicities. The Monte Carlo error in the posterior mean of 3 is less than 0.01 . Figure 1(b) shows posterior distributions of the more easily interpreted hazard ratio of placebo to drug treatment. These are readily obtained by transformation of the posterior samples of 3 .

The posterior mean of the baseline survival function and pointwise $90 \%$ limits for it are plotted in Figure 2. As $k$ increases, the shape approaches that of the exponential as chosen in the prior. Values of $k$ upto 10 give remarkably stable posteriors except in the right tail where a different $c(\cdot)$ function could be chosen to express higher prior uncertainty. In general, such stable ranges of $k$ will depend on the size of the data.

Figure 3 demonstrates the types of inference that can be easily obtained from the full posterior samples. In addition to the mean survival functions for the two groups, part (b) shorrs how all parameter uncertainty can be propagated to useful quantities such as the difference in the survival functions. A more detailed description at $t=10$ weeks is shown in part (c). It clearly conveys the effectiveness of the drug in maintaining remission past 10 weeks.

The calculations for this analysis were performed on a Hewlett-Packard 9000 Series 700 workstation using code written in SAS-IML. Generation of 5000 posterior samples was accomplished in 28 minutes. Code in FORTRAN or $\mathrm{C}$ could be expected to speed this up considerably. Convergence diagnostics not reported here showed a burn-in of 1000 would suffice. 


\section{Conclusion}

A semiparametric model using C'ox regression and the beta process was described and exemplified. The complex form of the posterior distribution. following latent variable transformations. Was shown to reduce to a computational model that conld be sampled easily: The beta process is a rich family of prior clistributions on the space of cumulatire hazards with easy to interpret parameters. However, in some contexts. it may be necessary or easier to model the hazard rate nomparametrically. One class of prior distributions for hazard rates is called the extended gamma process (Dvistra and Laud. 1981). A semiparametric analysis similar to the one in this paper for increasing. decreasing and L-shaped hazard rates can be developed. In another direction. the methods of this paper can be adapted for use with various frailty models. These developments will be reported elsewhere.

\section{ACKNOWLEDGEMENTS}

We thank Vils Hjort for personal communication clarifying the distribution of jumps in the beta process and Craig C'umbus for SAS-IMIL code for the Crilks-Wild algorithm. Research reported here was partially funded by a grant from the National Science Fonndation.

\section{APPENDIX}

\section{Beta Process Summary}

With $\operatorname{Beta}(\alpha, 3)$ denoting the beta distribution proportional to $x^{\alpha-1}(1-x)^{\beta-1}$ on $[0,1]$. we have.

Definition: Let $A_{0}$ be a cumulative hazard function with a finite number of jumps taking place at $t_{1}, t_{2}, \cdots, t_{m}$ and let $c(\cdot)$ be a piecewise continuous, nonnegative function on $[0, x)$. A process $A$ is called a beta process with parameters $c(\cdot)$ and $A_{0}(\cdot)$, denoted

$$
A \sim \operatorname{beta}\left(c(\cdot), A_{0}(\cdot)\right)
$$

if it has Lévy representation

$$
E\left(e^{-\theta \cdot A(t)}\right)=\left[\prod_{j: t_{j} \leq t} E\left(e^{-\theta S_{j}}\right)\right] \exp \left\{-\int_{0}^{1}\left(1-e^{-\theta s}\right) d L_{t}(s)\right\}
$$


with

$$
S_{j}=A\left\{t_{j}\right\} \sim \operatorname{Beta}\left(c\left(t_{j}\right) \cdot A_{0}\left\{t_{j}\right\} \cdot c\left(t_{j}\right)\left(1-. A_{0}\left\{t_{j}\right\}\right)\right)
$$

and

$$
d L_{t}(s)=\int_{0}^{t}\left[c(z) s^{-1}(1-s)^{c(z)-1} d / A_{0 . c}(z)\right] d s
$$

for $t \geq 0$ and $0<s<1$. where $A_{0 . c}(t)=A_{0}(t)-\sum_{t_{j} \leq t} t_{0}\left\{t_{j}\right\}$.

$t_{0}$ is the prior guess at the cumulative hazard. and $c(t)$ can be interpreted as the number at risk at $t$ in an imagined prior sample. The abore definition implies that the beta process has independent increments and at fixed points of discontinuity the increments have beta distributions. More general choices of $d L_{t}(s)$ are possible with attendant results given in Hjort (1990) and simulation techniques in Damien et al.(1996).

Let $X_{1}, \ldots, X_{n}$ be independent and identically distributed with cumulative hazard function $A$, and $A \sim \operatorname{bet} a\left(c(\cdot), A_{0}(\cdot)\right)$. Suppose $\left(T_{1}, \delta_{1}\right), \cdots,\left(T_{n}, \delta_{n}\right)$ is observed. where $T_{i}=\min \left(\mathrm{X}_{i}, c_{i}\right), \delta_{i}=I\left\{X_{i} \leq c_{i}\right\}$ and $c_{1}, \cdots c_{n}$ are censoring times. Define the counting process $N$ and the left-continuous at :isk process $Y$ as

$$
V(t)=\sum_{i=1}^{n} I\left\{T_{i} \leq t \& \delta_{i}=1\right\}, \quad Y(t)=\sum_{i=1}^{n} I\left\{T_{i} \geq t\right\},
$$

where $I$ is the indicator function. Then

$$
. t \mid\left(T_{1}, \delta_{1}\right), \cdots,\left(T_{n}, \delta_{n}\right) \sim \operatorname{beta}\left\{c(\cdot)+Y(\cdot), \int_{0}^{(\cdot)} \frac{c(s) d A_{0}(s)+d \cdot V(s)}{c(s)+Y(s)}\right\} .
$$

The posterior process contains fixed points of discontinuity even if the prior does not. These extra points occur at observations with $\delta_{i}=1$. The distribution of any jump is given by

$$
A\{t\} \mid d a t a \sim \operatorname{Beta}\left(c(t) A_{0}\{t\}+d N(t), c(t)\left(1-A_{0}\{t\}\right)+Y(t)-d N(t)\right) .
$$

\section{REFERENCES}

Andersen, P.K., Borgan, O., Gill, R.D. and Keiding, N. (1993). Statistical Models Based on Counting Processes. New York: Springer-Verlag.

Besag, J. and Green. P.J. (1993). Spatial Statistics and Bayesian Computation. J. R. Statist. Soc. B 55, 25-3i.

Breslow, N.E. (19T4). Covariate Analysis of Censored Survival Data. Biometrics 30. 89-99. 
Cox. D.R. (19-2). Regression . Models and Life Tables (with Discussion). J. R. Stutist. Soc. B 34. 18i-220.

Damien. P.. Laud. P.IV.. and Smith. A.F.M.. (1995). Approximate Random Variate Generation from Infinitely Divisible Distributions with Applications to Bavesian Inference. J. R. Statist. Soc. B 57. j4i-j63.

Damien. P.. Laud. P.W.. and Smith. A.F.MI.. (1996) Implementation of Baresian Lonparametric Inference Based on Beta Processes. Scand. J. Statist. 23. 27-36.

Dykstra. R. L. and Laud. P. W. (1981). Bayesian Nonparametric Approach Toward Reliability, Ann. Statist. 9, 356-36i.

Efron. B. (19Ti). The Efficiency of Cox's Likelihood Function for C'ensored Data. J. Am. Statist. Assoc. 72, 55i-56.5.

Ferguson, T. S. (1973). A Bayesian Analysis of Some Nonparametric Problems. Ann. Statist. 1, 209-230.

Gilks, W.R. and Wild. P. (1992). Adaptive Rejection Sampling for Gibbs Sampling. Appl. Statist. 41, 33T-348.

Gill. R.D. and Johansen. S. (1990). A Survey of Product-Integration with a View toward Application in Survival Analysis. Ann. Statist. 18, 1501-1555.

Hjort, N.L. (1990). Nonparametric Bayes Estimators Based on Beta Processes in .lodels for Life History Data. Ann. Statist. 18, pp 1259-1294.

Kalbfleisch, J.D. (1978). Non-parametric Bayesian Analysis of Survival Time Data. J. R. Statist. Soc. B 40, 2, 214-221.

Kalbfleisch, J.D. and Prentice, R.L. (1980). The Statistical Analysis of Failure Time Data. New York: John Wiley.

Iilein. J.P. and Moeschberger. M.L. (1996). Survival Analysis: Methods for Censored and Truncated Data. New York: Springer-Verlag.

Smith. A.F.M. and Roberts, G.O. (1993). Bavesian Computations via the Gibbs Sampler and Related Markov Chain Monte Carlo Methods. J. R. Statist. Soc. B 55. $3-23$.

Walker, S.G. (1996). Random Variate Generation from an Infinitely Divisible Distribution via Gibbs Sampling. Technical Report, Imperial College, London. 


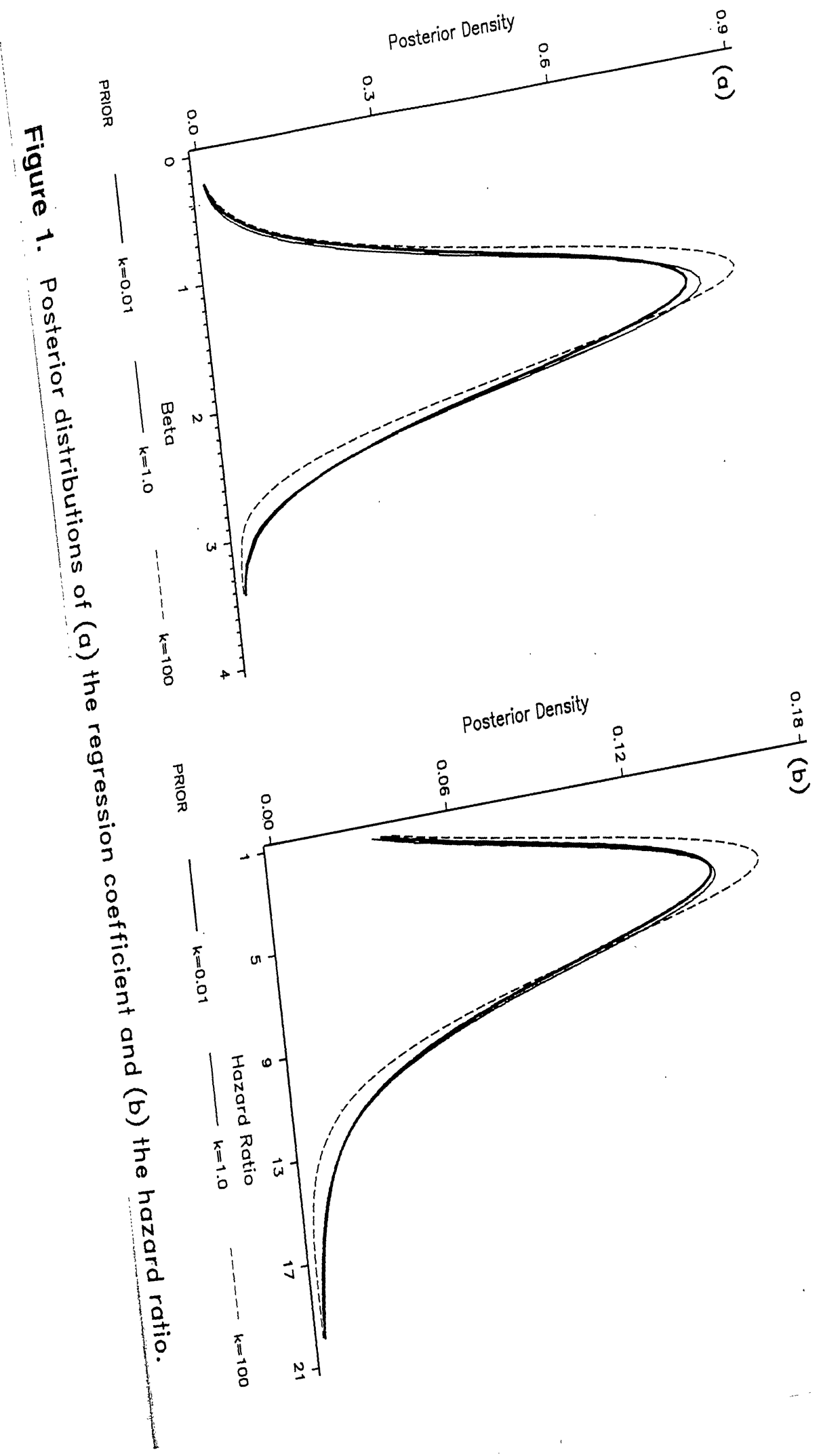



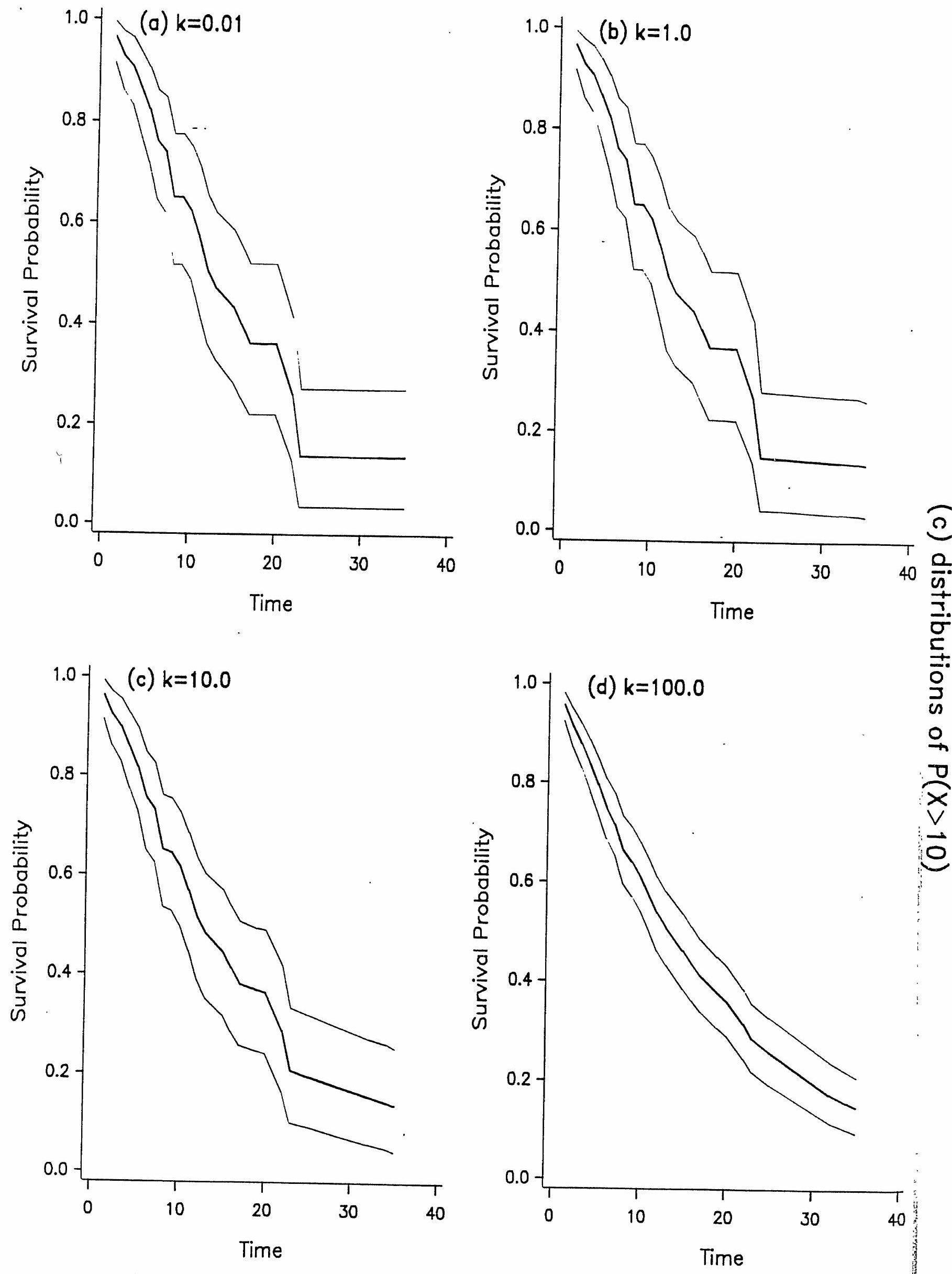

Figure 2. Posterior mean and central $90 \%$ limits for baseline survival function. 

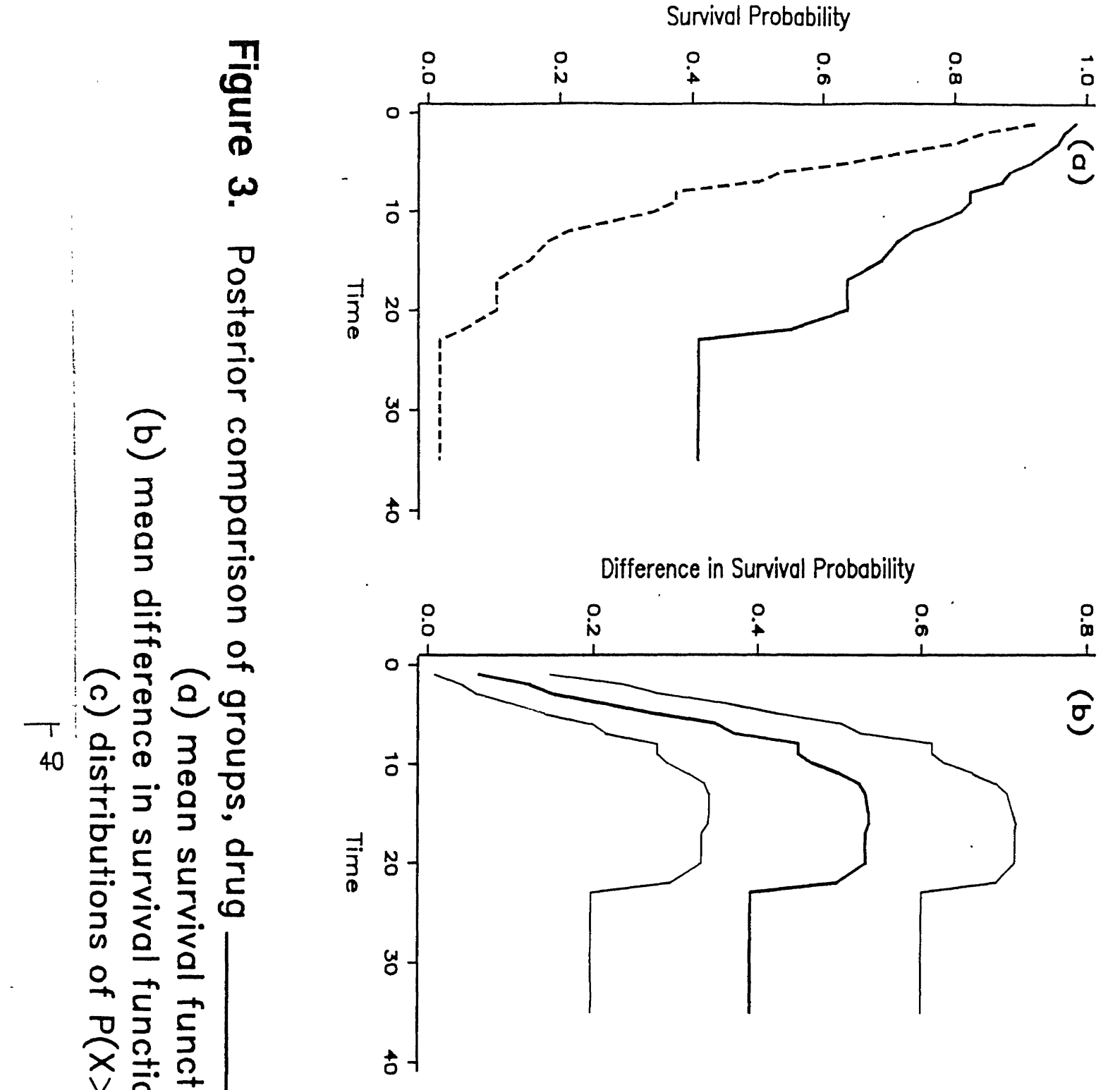

Posterior Density

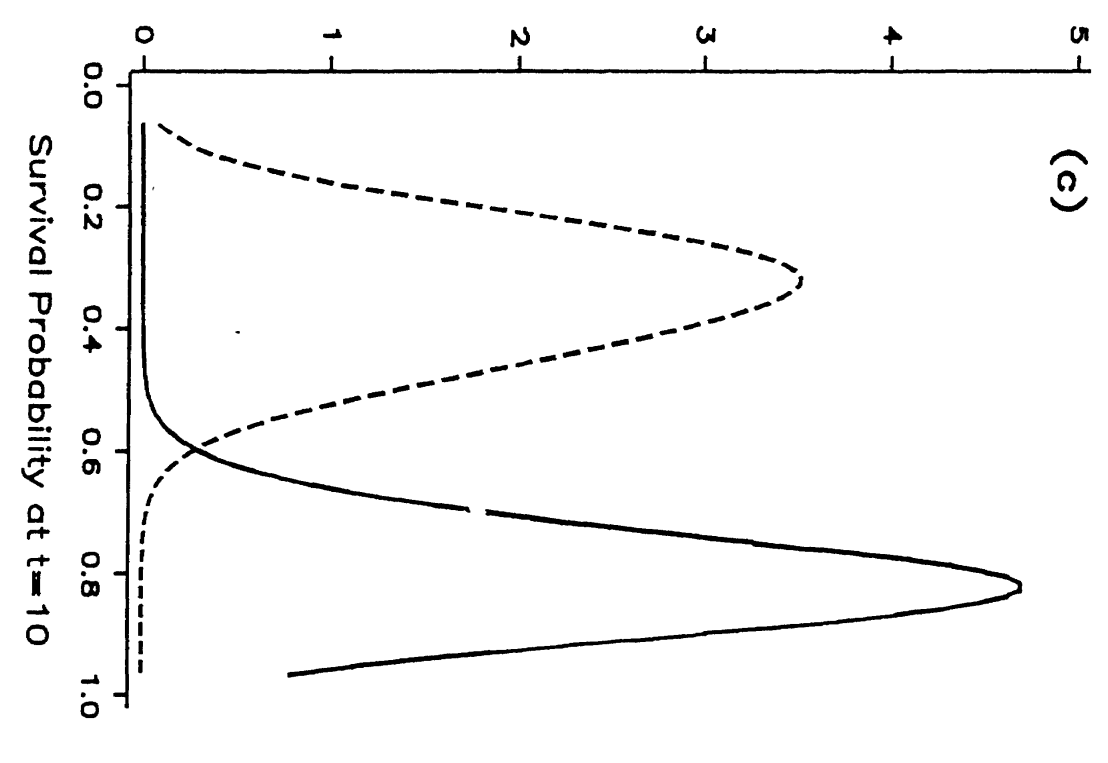

